\title{
Geometry- and diffraction-independent ionization probabilities in intense laser fields: probing atomic ionization mechanisms with effective intensity matching
}

\author{
W. A. Bryant S. L. Stebbings, E. M. L. English, T. R. J. Goodworth, and W. R. Newel $\dagger$ \\ Department of Physics and Astronomy, University College London, Gower Street, London WC1E 6BT, UK \\ J. McKenna, M. Suresh, B. Srigengan, and I. D. Williams \\ Department of Pure and Applied Physics, Queen's University Belfast, Belfast BT7 1NN, UK \\ I. C. E. Turcu, J. M. Smith, E. J. Divall, C. J. Hooker, and A. J. Langley \\ Central Laser Facility, Rutherford Appleton Laboratory, Chilton, Didcot, Oxon OX11 OQX, UK
}

(Dated: September 7, 2018)

\begin{abstract}
We report a novel experimental technique for the comparison of ionization processes in ultrafast laser pulses irrespective of pulse ellipticity. Multiple ionization of xenon by $50 \mathrm{fs} 790 \mathrm{~nm}$, linearly and circularly polarized laser pulses is observed over the intensity range $10 \mathrm{TW} / \mathrm{cm}^{2}$ to $10 \mathrm{PW} / \mathrm{cm}^{2}$ using Effective Intensity Matching (EIM), which is coupled with Intensity Selective Scanning (ISS) to recover the geometry-independent probability of ionization. Such measurements, made possible by quantifying diffraction effects in the laser focus, are compared directly to theoretical predictions of multiphoton, tunnel and field ionization, and a remarkable agreement demonstrated. EIM-ISS allows the straightforward quantification of the probability of recollision ionization in a linearly polarized laser pulse. Furthermore, probability of ionization is discussed in terms of the Keldysh adiabaticity parameter $\gamma$, and the influence of the precursor ionic states present in recollision ionization is observed for the first time.
\end{abstract}

PACS numbers: $32.80 . \mathrm{Rm}, 42.50 . \mathrm{Hz}, 42.65 . \mathrm{Sf}$

\section{INTRODUCTION}

Over the last decade and a half, the advent of modern laser technology has resulted in a fruition of significant experimental studies on the interaction of intense ultrafast laser pulses with dilute matter. The success of these works has naturally precipitated a reaction from the theoretical community, which has lead to a highly productive dialogue. Particular highlights are the observation [1] of high harmonic generation (HHG), the use of HHG to generate attosecond-timescale bursts of XUV radiation [2], nonsequential ionization of atoms [3] and molecules [4] through electron rescattering and the enhanced ionization of molecules leading to Coulomb explosion [5]. Experimental advances by the authors and co-workers have allowed the investigation of the interaction of ultrafast laser pulses with ionic targets, where the ionization of ground and metastable $\mathrm{Ar}^{+}[\underline{6}], \mathrm{C}^{+}$[7] and $\mathrm{Xe}^{+}[8]$ has been observed for the first time. Central to all of these studies is the observation of process which are dependent on focused laser intensity, traditionally facilitated by variation of the pulse energy. While being straightforward to carry out experimentally, this technique suffers the drawback that, as the pulse energy is varied, the size of the laser focus is altered. Such geometrydependent measurements are of course still of great importance, and have allowed major advances in the under-

\footnotetext{
*Electronic address: w.bryan@ucl.ac.uk
}

${ }^{\dagger}$ Electronic address: w.r.newell@ucl.ac.uk standing of laser-matter interactions: the drawback is in making comparisons with theory. A different approach has been facilitated by the ongoing development of laser amplification techniques [9], permitting macroscopic control of laser focal conditions through intensity selective scanning (ISS) 10, 11]. Here the laser focal geometry is constant, and a spatially selective detector is used to image different regions of the focus. Such measurements are of particular interest to the atomic physics community, with regards, for example, to the response of noble gas atoms to focused femtosecond laser pulses.

While a number of experimental studies have compared atomic ionization using circular and linear polarized laser pulses, for recent examples see [4, 12], to the authors' knowledge, all have employed the traditional geometry-dependent technique. For a particular pulse energy, there is a change in electric field amplitude when the polarization is switched from linear to circular, making a comparison problematic. We propose a new method to circumvent this problem, as a development of ISS. A constant pulse energy ratio between linear and circular polarizations is defined such that the relative ionization yield is constant as the laser focus is translated past the spatially selective detector, irrespective of laser polarization. This technique of effective intensity matching (EIM) coupled with ISS is employed to examine ultrafast strong field ionization of xenon: EIM-ISS results are presented.

Before quantitative analysis of the EIM-ISS results is possible, it is necessary to remove the geometry dependence inherent in the ionization yields. A method to perform such a deconvolution has been proposed in [10]. An 
analogous technique has been presented by the authors [13], where a numerical inversion is employed to remove the geometry dependence from the ISS results. However both techniques only allow the removal of Gaussian focusing. Modern laser systems typically generate pulses with a Gaussian profile in the far-field. However, optical transport systems impose geometrical constraints such that it is practically impossible to propagate this perfect profile into the experimental chamber. Such beam propagation has been addressed recently by Lü and coworkers 14] through the application of an aperture function approximation [15] to a multi-apertured optical system. Unfortunately, this approximation, even with little or no beam truncation, will introduce oscillatory distortions to the laser intensity. Zhang and co-workers 16. have presented an elegant analysis of the diffraction of a focused beam, with particular application to traditional geometry-dependent intensity variation measurements. In the present work, beam diffraction is quantified before focusing takes place: this treatment better representing typical experimental systems.

The spatial insensitivity of most instruments employed in intensity variation measurements 12, 17, 18, 19, 20, 21, 22] average over such diffraction effects, as all of the confocal volume lies within the volume that the instrument images. In the case of EIM-ISS, the very spatial sensitivity that makes the technique so powerful also makes it susceptible to optical distortions. Given that the EIM-ISS technique naturally discriminates between low-intensity large volume and high-intensity small volume processes, then before the laser - atom system under investigation can be fully understood, it is vital to quantify the spatial distribution of laser intensity. Herein, we present the results of an analytical treatment of the focusing of a truncated Gaussian laser pulse through an arbitrary ABCD (where A to D are elements of the System Matrix) optical system, the derivation of which is given in Appendix A.

By matching the optical conditions in our $\mathrm{ABCD}$ model to those in our experimental focus, we then remove the geometry dependence from the EIM-ISS data, revealing for the first time geometry-free and diffractionfree probabilities of ionization for both linear and circular polarizations, and compare them directly to theoretical predictions.

\section{APPLICATION TO LASER - DILUTE MATTER INTERACTIONS}

In the following section, the optical system employed is defined, then the xenon EIM-ISS data is presented. The apertured solution for an arbitrary ABCD optical system is then employed to remove the geometrical influence of the volume of the laser focus, resulting in geometryindependent atomic ionization probabilities. Ionization mechanisms are then discussed in detail. Finally, the variation of geometry-independent ionization probability

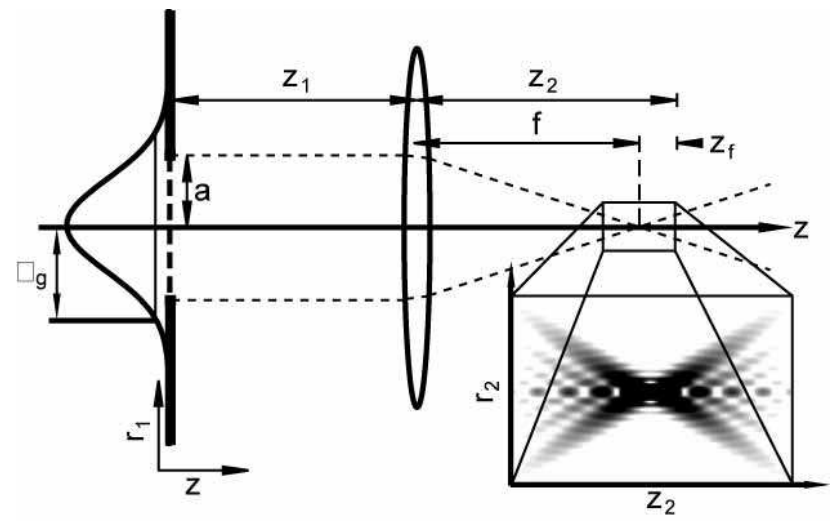

FIG. 1: Illustration of the cylindrically symmetric optical system employed in recent laser - dilute matter interactions. The aperture, radius $a$, lies in the input plane at $z=0$, with radial co-ordinate $r_{1}$. The resulting focus lies in the output plane at $z=z_{1}+z_{2}$, with radial co-ordinate $r_{2}$. The focal length of the lens is $f$, and $z_{f}=z_{2}-f$ is position with respect to the focus.

with the Keldysh parameter 23] is presented, allowing ionization mechanisms to be discussed in terms of the relative frequencies of the tunneling electron and the laser field.

\section{A. Optical system}

In recent experimental studies published by the authors and co-workers [6, 7, 8, 13] the optical system is as illustrated in Fig. 1] with a corresponding system matrix

$$
\left(\begin{array}{ll}
A & B \\
C & D
\end{array}\right)=\left(\begin{array}{cc}
1-\left(z_{2} / f\right) & z_{1}+\left(1-z_{1} / f\right) z_{2} \\
-1 / f & 1-\left(z_{1} / f\right)
\end{array}\right)
$$

where $z_{1}$ and $z_{2}$ correspond to the distance between the aperture and lens, and lens and focus, and $f$ is the focal length of the lens. We also define $z_{f}=z_{2}-f$ as the position parallel to the beam propagation direction with respect to the focus. The system matrix is derived using straightforward matrix optics, and is the transformation from the input plane (containing the aperture) to the output plane, through a translation, a refraction and a second translation to the focus. In the present work, the following values apply: $z_{1}=300 \mathrm{~mm}, z_{2}=250 \mathrm{~mm}, f$ $=250 \mathrm{~mm}$ and the aperture radius $a=11 \mathrm{~mm}$. Given the wavelength, $\lambda=790 \mathrm{~nm}$, the only quantity which is unknown is the beam radius $\omega_{g}$ before the aperture at which diffraction occurs. The fixed aperture, located at $z=0$, defines the reference plane for the System Matrix. As presented in Appendix A, we derive a solution for the laser intensity distribution in the vicinity of the focus, which accounts for diffraction of the incoming laser beam at an aperture of finite diameter. Such a solution is particularly applicable to ultrafast (Ti: sapphire based) 
physics, where the intensity distribution in the laser focus is rarely Gaussian. Fig. 2(a)-(j) shows the result of simulating the focus of the current optical system for the fixed radius aperture and a range of beam radii. Initially, the beam radius $\omega_{g}=5 \mathrm{~mm}$, and the focus created is almost identical to the unapertured form described by Eq. (A6) in Appendix A. However, as the beam radius is increased, the focus is disturbed by the diffraction of the incoming beam at the aperture. As the beam radius becomes comparable to the aperture radius, the focus shows pronounced lines of maximum and minimum intensity in both $z_{f}$ and $r_{2}$. The isointensity contours in Fig. 22(a)(j), separated by an order of magnitude, illustrate that as the beam radius is increased, the focal spot size increases along $r_{2}$ contrary to the behaviour expected in the case of an unapertured Gaussian beam.

\section{B. Effective intensity matching (EIM) as applied to ISS}

The experimental apparatus employed in the present work has been described in detail [6, 7, 8, 13, 24], so only a brief outline is given here. The $20 \mathrm{~mJ}, 790 \mathrm{~nm}$ 50 fs output of the ASTRA Ti:sapphire laser is transmission focused on to the target gas in the source region of the time-of-flight mass spectrometer. Given the high power of the laser used in our recent work, intensities in excess of $10^{17} \mathrm{Wcm}^{-2}$ are routinely generated in a long focus. Indeed, the intensity range required for ultrafast ionization rate studies, typically $>10^{13} \mathrm{Wcm}^{-2}$ is produced $\approx 10 \mathrm{~mm}$ from the centre of the focus. The 250 $\mu \mathrm{m}$ entrance aperture located in the time-of-flight mass spectrometer places a tight spatial limitation on the volume of the focused laser beam which produces ion signal from the target gas, and by translating the focusing lens parallel to the $\mathrm{z}$-axis, the spectrometer is exposed to different regions of the focus. Typically, such ISS or z-scan experiments are performed by averaging the ion signal measured at each lens position, $z_{f}$.

To explore the ionization mechanism in xenon we have developed a novel experimental technique, namely effective intensity matching, combined with intensity selective scanning (EIM-ISS). The essence of EIM-ISS is to define a constant ratio, $R_{\mathrm{EIM}}$ between the laser intensities of the linearly $\left(I_{\text {lin }}\right)$ and circularly $\left(I_{\text {circ }}\right)$ polarized laser beams such that the spatial distribution of the ions detected for each polarization are the same for all $z_{f}$ values assuming that nonsequential ionization processes are negligible. Before recording the xenon data presented, the ionization of neon was observed with circularly and linearly polarized radiation to define the ratio $R_{\mathrm{EIM}}$. Neon is a good test gas as, of all the noble gases, it is least susceptible to nonsequential (recollision) ionization [25]. Our studies, not presented here [8], reveal that $R_{\mathrm{EIM}}=$ $I_{\text {lin }} / I_{\text {circ }}=0.65 \pm 0.02$ gives a remarkable match in the $\mathrm{Ne}^{+}$ionization signal over a large $(\approx 10 \mathrm{~mm})$ range of $z_{f}$, equivalent to an intensity range $10^{13} \mathrm{Wcm}^{-2}$ to

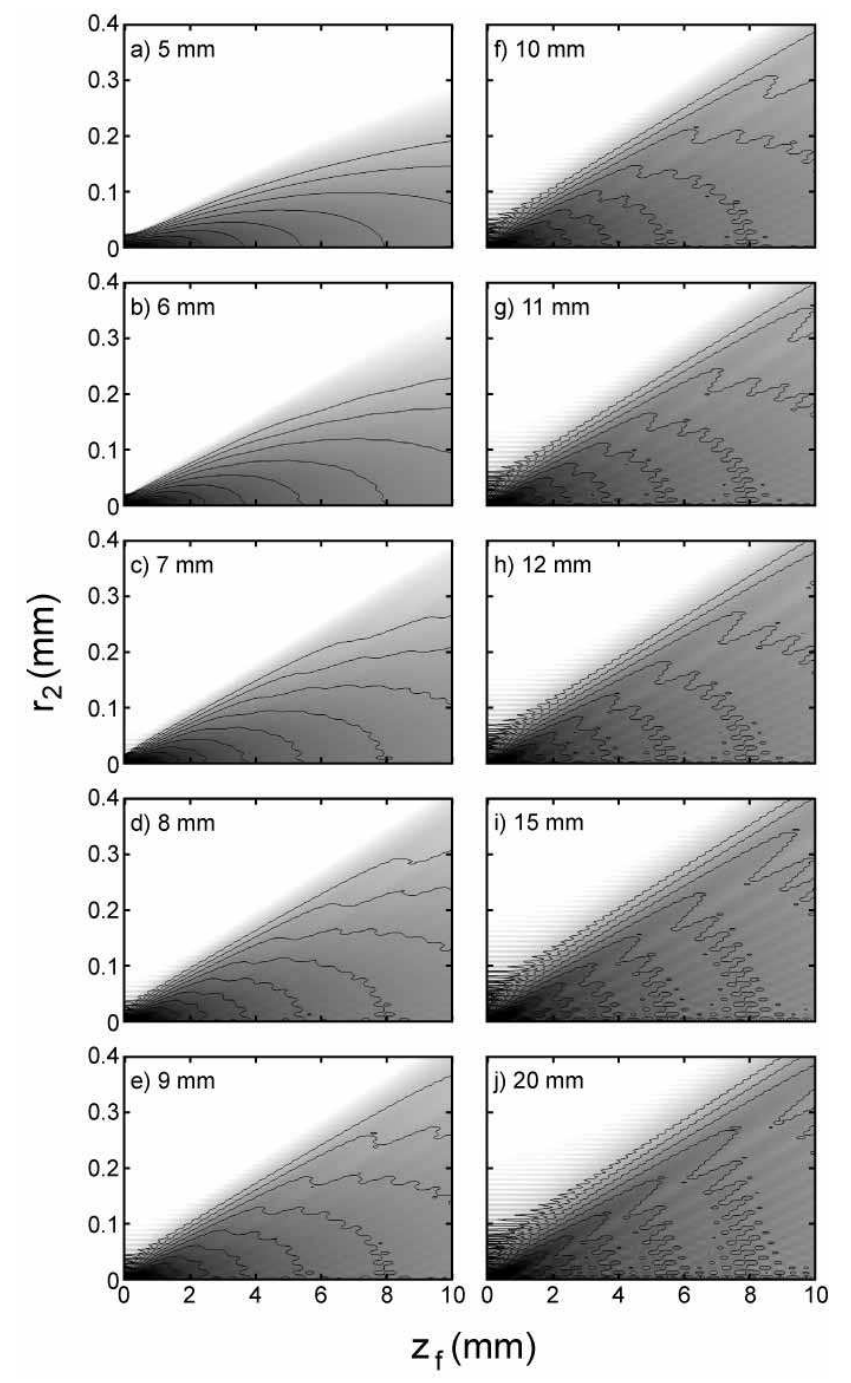

FIG. 2: (a) to (j) Simulated intensity distributions in logarithmic grayscale with isointensity contours, separated by an order of magnitude, for the optical system in Fig. 1. The aperture radius $a=11 \mathrm{~mm}$ is kept constant, while the beam radius $\omega_{g}$ is varied from 5 to $20 \mathrm{~mm}$. Diffraction is clearly apparent even when the aperture is considerably larger than the beam radius.

$10^{16} \mathrm{Wcm}^{-2}$. A similar approach was reported in 26. to explain threshold ionization intensities observed with linearly and circularly polarized light in a long-pulse (1 ps) traditional intensity variation measurement. However, the present study and that of 8] are of a higher precision, being the culmination of a systematic investigation, and are unique in the area of ultrafast intense field interactions.

The exact value of $R_{\mathrm{EIM}}$ is determined by the influence of the different laser polarizations and electric field amplitudes on the ionization process. In the linearly polarized case, the sinusoidal laser electric field is modulated by the pulse envelope: the field amplitude oscillates under a typically $\operatorname{sech}^{2}$ temporal profile. With circular 


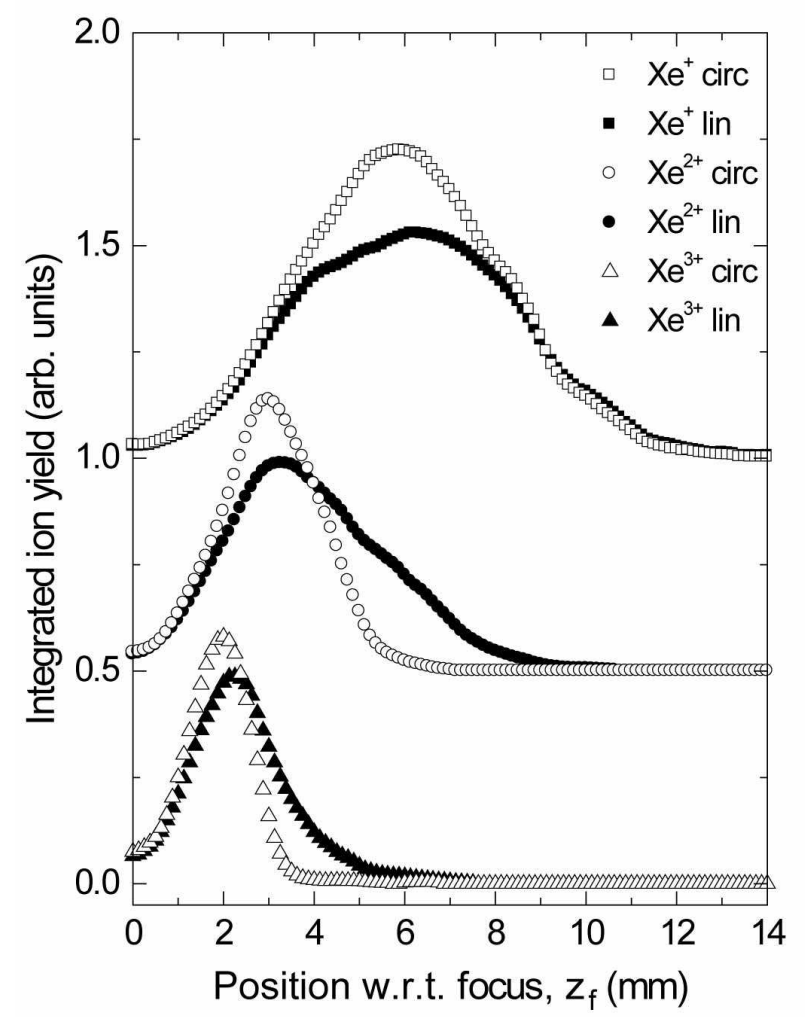

FIG. 3: Raw EIM-ISS data for the ionization of xenon to $\mathrm{Xe}^{n+}(n=1,2,3)$. The integrated xenon ion yield is recorded as function of focusing optic position with respect to the axis of the spectrometer, in Fig. 1 equivilant to $z_{f}$. Linear (solid symbols) and circular (open symbols) polarized laser pulses are employed, where EIM is used to define the distribution of ion signal with varying $z_{f}$. The presence of nonsequential (recollision) ionization is apparent in the $\mathrm{Xe}^{2+}$ and $\mathrm{Xe}^{3+}$ signal as signal enhancement at low intensity (large $z_{f}$ ) in the case of linear polarization. Ripples in the ion yield indicate the presence of diffraction.

polarization the electric field is continually present, and the electric field direction rotates through $2 \pi$ during the laser period, and the field amplitude typically takes a $\mathrm{sech}^{2}$ profile. In the circularly polarized field, the projection of the angular momentum imparted to the electron is conserved along the direction of beam propagation. However, in the linearly polarized field, momentum is imparted to the electron in the direction of electric field oscillation, i.e. perpendicular to the propagation axis. This has an important influence on tunnel ionization, as recently noted by Tulenko and Zon [27] in which the rate of tunnelling depends on the magnetic quantum number of the tunnelling electron: in 28 it was concluded that the ionization rate should be identical, irrespective of polarization type.

With $R_{\text {EIM }}$ defined, the spectrometer source region was filled with xenon to a pressure of $1 \times 10^{-8}$ mbar: a low pressure is used to avoid the influence of space-charge effects. All ions generated were averaged over 500 laser shots for each position along $z_{f}$. The observed $z$-scan is presented in Fig. 3] showing $\mathrm{Xe}^{n+}(n=1,2,3)$ product ion yields, with the $\mathrm{Xe}^{2+}$ and $\mathrm{Xe}^{+}$yields displaced vertically by 0.5 and 1.0 respectively for clarity. By limiting the field of view of the spectrometer, the ion signal from a particular charge state at each z-position is an integral over all $r_{2}$ and a narrow range of $z_{f}$. This selectivity is apparent in the data in Fig. 3 Starting at the focus, each ion yield presented is observed to increase from near zero to a maximum, the position of the maximum moving to lower $z_{f}$ with increasing charge state, $n$. Each successive charge state requires an increase in intensity to maximize its ionization yield, a common result of traditional intensity measurements 12, 17, 18, 19, 20, 21, 22. . Subtle ripples present in the data presented in Fig. 3 are the result of diffraction in the ultrafast laser focus. Importantly, linear and circular time-of-flight spectra are recorded at each $z_{f}$ position as the focusing optic is translated, rather than recording a linear scan followed by a circular scan. This ensures that conditions within both the spectrometer and laser focus undergo minimal variation.

Xenon has a far greater propensity for ionization through recollision in a linear laser field than neon, giving rise to the major differences between linearly and circularly polarized light observed in the EIM-ISS data in Fig. [3 The data recorded with circular polarization is recollision-free, as the probability of the first-ionized electron returning to the ion in a circularly polarized field is negligible 29]. The 'signature' of recollision is clearly apparent in the $\mathrm{Xe}^{2+}$ and $\mathrm{Xe}^{3+}$ data as an enhancement of the integrated ion yield with linear polarization at positions of $z$ at larger distances from the focus than the maxima (for example, in $\mathrm{Xe}^{2+} 4.5 \mathrm{~mm}<z_{f}<8 \mathrm{~mm}$ ). This enhancement is due to recollision ionization in the linear field being more efficient than sequential multiple ionization by the laser field at low intensities (i.e. large $z_{f}$ with respect to the centre of the focus).

The enhancement of ion yield by recollision ionization in $\mathrm{Xe}^{2+}$ and $\mathrm{Xe}^{3+}$ is directly responsible for the significant differences in the $\mathrm{Xe}^{+}$yield curves. This is a consequence of the conservation of confocal volume, necessitated by the following important points. The core result of EIM-ISS is that the effective laser intensity is identical for linear and circular polarizations. Given that the number density is constant, the ionization yield from sequential ionization of the atoms in the confocal volume is made to match for both polarizations over all $z_{f}$. However, the volume of the laser focus must be conserved, therefore, we observe for the first time the true interplay between the volumes generating a charge state $\mathrm{Xe}^{n+}(n=$ $1,2,3 \ldots)$, which totally contains and often overlaps with the volume generating all higher charge states. Therefore the suppression of integrated ion yield in the case of $\mathrm{Xe}^{+}$ in the range $3 \mathrm{~mm}<z_{f}<8 \mathrm{~mm}$ is due to the depopulation of the $\mathrm{Xe}^{+}$volume in the laser focus (when the laser is linearly polarized) through the observed mechanism of recollision ionization to higher charge states. An analo- 
gous depletion is observed around the maximum of the $\mathrm{Xe}^{2+}$ yield, caused by depopulation of the $\mathrm{Xe}^{2+}$ volume by recollision ionization to $\mathrm{Xe}^{3+}$ in linearly polarized radiation. This sequence is repeated for the higher charge states. By comparing the sum of ion yields over all charge states at each $z$ for the two polarizations, a near exact match is observed, confirming conservation of focal volume. This then is the major benefit of EIM-ISS, allowing direct comparisons to be made between atomic ionization mechanisms for the first time.

\section{Deconvolution of EIM-ISS results: volume-independent ionization probabilities}

To further develop our understanding of the interplay of ionization mechanisms as observed using EIM-ISS using both linear and circular polarizations, we turn now to removing the geometry dependence of the ionization signal from the EIM-ISS data in Fig. 3] The aim of such analysis is the recovery of geometry-independent ionization probabilities.

Van Woerkom and co-workers have previously established a technique for the removal of the dependence of ionization probability on focal volume 10, 11, where the ionization probability $\Omega(I)$, may be calculated from the $z_{f}$ dependent ion signal $S\left(z_{f}\right)$ via the on-axis intensity distribution $I_{a x}\left(z_{f}\right)$ according to:

$$
\Omega\left(I_{a x}\left(z_{f}\right)\right) \propto\left(\frac{I_{a x}\left(z_{f}\right)}{d I_{a x}\left(z_{f}\right) / d z_{f}}\right) \frac{d}{d z_{f}}\left[I_{a x}\left(z_{f}\right) S\left(z_{f}\right)\right]
$$

In the present work, we have refined this treatment, firstly to allow for the non-Gaussian nature of the focussed beam and secondly removing the dependence of ionization probability on the intensity distribution within the laser focus, despite the presence of diffraction. The latter is achieved by applying Eq. (A9) in Appendix A to Eq. (2) where $I_{a x}=\left|U\left(r_{2}=0, z_{f}\right)^{2}\right|$.

Although the $1 / \mathrm{e}^{2}$ beam radius is difficult to define accurately, it is of a comparable size to the final aperture radius. Furthermore, the ionization signal of the first three charge states is expected to saturate within the range of $z_{f}$ (i.e. intensity) in the current experiment 21], thus the gradient of the ionization probability should tend to zero at high intensity for all change states, $n$. At intensities above saturation, it is reasonable to expect ionization probability to decrease, as ionization to higher charge states becomes increasingly more likely. However, if we define some saturation intensity $I_{\mathrm{SAT}}$, which occurs at a corresponding on-axis distance $z_{\mathrm{SAT}}$ from the focus, at $z_{f}<z_{\mathrm{SAT}}$ (i.e. on-axis $I>I_{\mathrm{SAT}}$ ), $I_{\mathrm{SAT}}$ is still present off-axis thus occupying a larger proportion of the volume than the on-axis contribution. The result of applying Eq. (2) is only valid at intensities $<I_{\mathrm{SAT}}$ : at intensities $>I_{\mathrm{SAT}}$, we define the probability of ionization as unity. This volume- and diffraction-free intensity dependent quantity is referred to as the partial probability

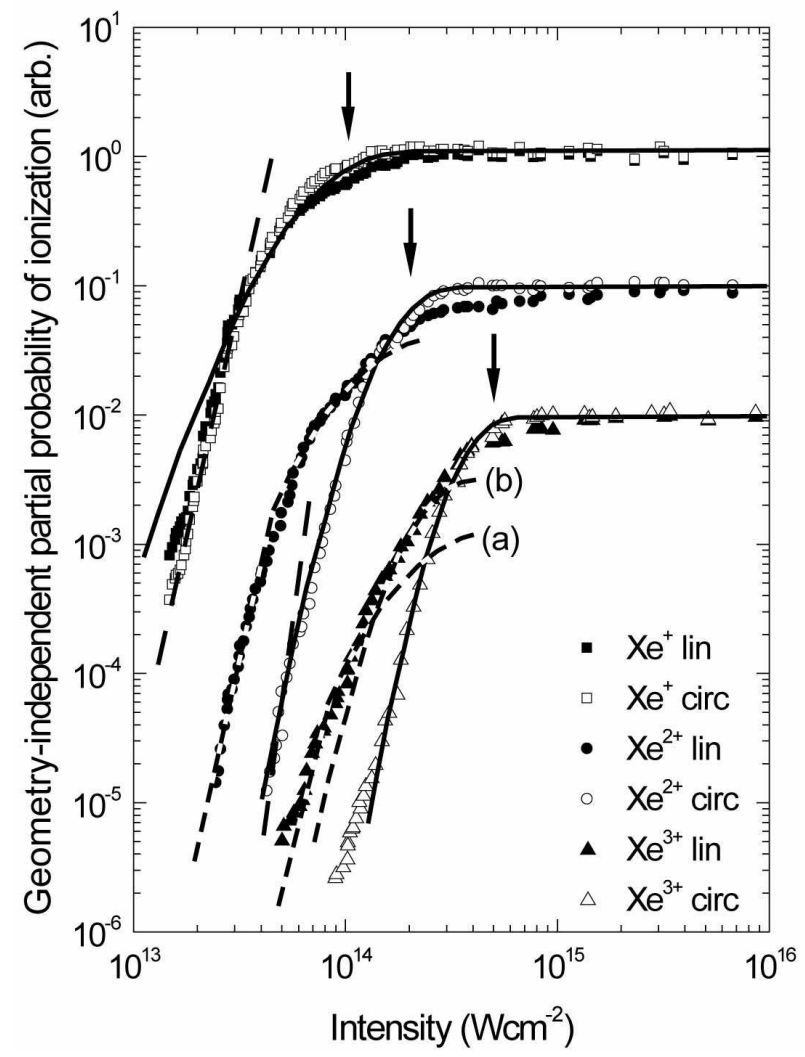

FIG. 4: EIM geometry-independent partial probability of ionization (PPI) for $\mathrm{Xe}^{n+}(n=1,2,3)$ as function of intensity, measured for both linear (solid symbols) and circular (open symbols) polarizations. Increasing charge states are normalized to descending orders of magnitude for clarity. Three types of sequential ionization are identified: multiphoton (low intensity, constant gradient, long dashed line), tunnelling (intermediate intensity, decreasing gradient, solid line) and field ionization (high intensity, vertical arrow). Recollision ionization (short dashed line) is also observed with linear polarization. See text for discussion.

of ionization (PPI). If the conserved probability of ionization $(\mathrm{CPI})$ is required, this may be readily calculated according to CPI $(n)=$ PPI $(n)$ - PPI $(n+1)$, where $n$ is charge state, as before. This technique will be applied in future publications.

The geometry- and diffraction-independent PPIs are now recovered from the EIM-ISS data in Fig. 3 and are presented in Fig. 团 where the saturated (unity) ionization probabilities of the increasing charge states are normalized to descending orders of magnitude to aid visual presentation. The beam radius $\omega_{g}$ is estimated from a measurement of the unfocused beam profile, and small adjustments (of the order of $0.1 \mathrm{~mm}$ ) made to $\omega_{g}$ until the PPI curves recovered do not exhibit rapid changes of gradient with intensity. The beam radius $\omega_{g}=10.25 \mathrm{~mm}$ used to recover these ionization probabilities is found to be not only consistent over all charge states observed in xenon $(n=1,2,3)$, but also for a wide range of atomic 
(the other Noble gases) and molecular $\left(\mathrm{H}_{2}, \mathrm{D}_{2}, \mathrm{~N}_{2}, \mathrm{CO}_{2}\right)$ targets covered in our studies.

To quantify the results presented in Fig. 4, the intensity-dependent probability of ionization to $\mathrm{Xe}^{n+}(n$ $=1,2,3)$ by four ionization mechanisms is now discussed. Starting with the lowest intensity, the following mechanisms are apparent in Fig. 目

(i) Multiphoton ionization (MPI) is predicted by lowest-order perturbation theory (LOPT) to vary according to $I^{N}$ [30], where $N$ is the number of $790 \mathrm{~nm}(1.56$ $\mathrm{eV})$ photons absorbed, thus on a log-log plot of probability vs. intensity a constant gradient of $N$ should exist. Increasing charge state $n$ requires an increasing number of photons. For example, $\mathrm{Xe}^{2+}$ production requires an energy of $21.21 \mathrm{eV}$, therefore the absorption of at least fourteen $790 \mathrm{~nm}$ photons is required. The expected gradients for MPI of $\mathrm{Xe}^{n+}$ are $7.8(n=1), 13.6(n=2)$ and $20.6(n=3)$. For $n=1$ and 2, the expected gradients are shown in Fig. 4 as long dashed lines. For $n=$ 3 , there is no MPI observed within the sensitivity of the experiment, determined by the low target gas density.

(ii) Tunnel ionization (TI), as described initially by Keldysh 23] and later refined by Popov and co-workers 28], is the result of a bound electron tunnelling out of the atom through the laser-modified Coulomb potential. With increasing intensity, TI becomes more efficient, as the laser + Coulomb barrier width decreases. Represented in Fig. 4 as solid lines, Keldysh theory predicts a tunnel rate, which we have converted to probability. Note that the amount of TI observed is not always wellpredicted by tunnelling theory, as observed recently by Yamakawa and co-workers 31] who introduced scaling factors in order to match theory and experiment. In the present work, when the TI theory is shifted to improve the fit with the PPI, the factors used are made clear.

(iii) At the highest intensities, classical field ionization (FI) dominates, as the electric field of the laser pulse is sufficient to rapidly suppress the Coulomb potential, allowing direct liberation of the electron. The intensity at which FI is predicted to occur is indicated by the vertical arrows in Fig. 4, calculated using the over-the-barrier model. The intensity at which FI dominates should coincide with the saturation of TI.

Ionization mechanisms (i) to (iii) are sequential in nature, and due to the EIM technique should occur at the same effective laser intensity for linear and circular polarizations (evident from the overlap of the $\mathrm{Xe}^{+}$PPIs in Fig. 4). As the ionization potential increases with charge state, we expect a systematic increase in the intensity required to produce a certain ionization mechanism. Furthermore, as discussed with reference to Fig. 3, there is an enhancement of signal in a linearly polarized laser pulse at low intensities, thus we must also consider nonsequential ionization processes:

(iv) Recollision ionization (RI) 29], also referred to as nonsequential double or multiple ionization (NSDI or NSMI), predominantly occurs due to the linear laser field driving a correlated liberated electron back to the par- ent ion, thus initiating secondary ionization 32]. This process has been the subject of a number of COLTRIMS studies 33. In Fig. 4, the enhancement of $\mathrm{Xe}^{2+}$ and $\mathrm{Xe}^{3+}$ by RI is clearly seen in the difference of PPI curves for linear and circularly polarized radiation. In Fig. 4, the presence of RI is indicated by the short dashed lines, generated by transferring the theoretical prediction of sequential ionization of previous charge state(s) onto the linearly polarized data. For $\mathrm{Xe}^{2+} \mathrm{RI}$, the combined MPI and TI to $\mathrm{Xe}^{+}$PPI is fitted, whereas for $\mathrm{Xe}^{3+} \mathrm{RI}$, the MPI and TI to $\mathrm{Xe}^{+}$and the TI to $\mathrm{Xe}^{2+}$ is fitted as there is more than one recollision mechanism possible resulting in triple ionization.

By comparing the quality of fit of MPI, TI, FI and RI to the data in Fig. 4, we can directly determine how successfully the geometry- and diffraction-independent PPIs are predicted by theory. As the PPI measured with circular polarization is not influenced by RI, the circular data is therefore a more direct comparison to the predicted MPI and TI response.

For ionization to $\mathrm{Xe}^{+}$, MPI with the absorption of eight photons occurs at the lowest intensities $(<2.5 \times$ $10^{13} \mathrm{Wcm}^{-2}$ ) present in Fig. 4, indicating that LOPT is applicable. As the laser intensity increases, the PPI response tends away from MPI: the TI prediction fits the data excellently (i.e. directly predicted by Keldysh theory), even at intensities greater than that where FI is predicted to be the dominant mechanism. The $\mathrm{Xe}^{+}$ data clearly illustrates the intensity ranges over which the different ionization mechanisms apply.

When the laser field generates $\mathrm{Xe}^{2+}$, the absorption of fourteen photons is required for MPI to proceed. As is apparent from the long dashed line, only the very lowest intensities are predicted by MPI. TI theory is even more successful in this, accurately predicting a three order of magnitude increase in the PPI to better than 10 $\%$. However, it is necessary to translate the data by a factor of 0.91 in intensity to achieve this fit (cf 0.85 from 31], albeit during a shorter duration laser pulse, and geometry-dependent ion yield was measured). As with $n$ $=1, \mathrm{FI}$ is only reasonably accurate. In the case of the linearly polarized laser pulse, at low intensity, there is considerable RI present, as indicated by the short dashed line. The RI contribution follows clearly the shape of the theoretical prediction of sequential MPI and TI production (short dashed line) visually fitted to the $\mathrm{Xe}^{2+}$ data. This is consistent with $\mathrm{Xe}^{+}$being the source atom for $\mathrm{RI}$ to $\mathrm{Xe}^{2+}$. Such a method has been used by a number of groups (for example, see [21]) fitting RI in geometrydependent ion yield measurements, and it appears to be successful here.

LOPT does not apply at all in the case of $\mathrm{Xe}^{3+}$ as the expected gradient of 20.6 is far too high to be supported by the data. However, TI theory is able to accurately predict the PPI from low intensity right up to saturation, with the data translated by a factor of 0.85 in intensity, (cf 0.70 from 31]), here well-defined by FI (vertical arrow). There are now three possible nonsequential RI 
routes possible: (a) $0 \rightarrow 1 \Rightarrow 3$, (b) $0 \Rightarrow 2 \rightarrow 3$ and (c) $0 \Rightarrow 3$, where $\rightarrow$ indicates sequential ionization, $\Rightarrow$ indicates double ionization through $\mathrm{RI}$, and $\Rightarrow$ indicates triple ionization through RI. While there is strong evidence for mechanisms (a) and (b) as is clear from the two short dashed lines on Fig. 4, there is little evidence for the presence of mechanism (c). If it does occur, it is with a greatly suppressed PPI as compared to (a) and (b). The shape of the RI PPI for $n=3$ is well described by a combination of the theoretical prediction of sequential ionization to $\mathrm{Xe}^{+}$and $\mathrm{Xe}^{2+}$ as denoted by the short dashed lines (a) and (b) in Fig. 4 respectively.

The ease with which ionization mechanism may be determined from Fig. 4 is illustrated by considering what happens to a group of atoms in a circularly polarized laser field at an intensity of $2 \times 10^{14} \mathrm{Wcm}^{-2}$. Such an intensity will at least triply ionize the atom: all atoms will be ionized to $\mathrm{Xe}^{+}$by FI, between $40 \%$ and $50 \%$ of these ions will be further ionized to $\mathrm{Xe}^{2+}$ by TI, and of these ions, $1 \%$ will undergo MPI to $\mathrm{Xe}^{3+}$. The situation with linear polarization is complicated by NSMI, however the contribution is readily calculable from Fig. 4. Through a combination of multiphoton, tunnel and field ionization theory, the response of xenon to the ultrafast laser field may be accurately quantified.

\section{Ionization mechanism and the Keldysh parameter}

The pioneering theoretical work of Keldysh [23] established a rule for distinguishing between ionization mechanisms in a laser field using the fact that the ionization is governed by the relative frequency of the laser field and the tunnel frequency of the electron. The ratio of these frequencies is defined as the adiabaticity, or Keldysh parameter, $\gamma$ [23, 28], and allows the ionization mechanism to be broadly determined:

$$
\begin{array}{lll}
\gamma=\frac{\omega_{\text {laser }}}{\omega_{\text {tunnel }}}=\sqrt{\frac{E_{i}}{2 U_{p}}} & & \text { where } \\
\gamma \gg 1 & \text { MPI } \\
\gamma \simeq 1 & \text { TI } \\
\gamma \ll 1 & \text { FI }
\end{array}
$$

In Eq. (3), $U_{p}=9.33 \times 10^{-14} I \lambda^{2}$ is the pondermotive potential of the laser field, with intensity $I$ in $\mathrm{Wcm}^{-2}$ and laser wavelength $\lambda$ in $\mu \mathrm{m}$, and $E_{i}$ is the ionization potential of the atom in $\mathrm{eV}$. The accepted definition is that when $\gamma \gg 1$, the frequency of the laser is greater than the tunnelling frequency, hence MPI results. When $\gamma \simeq 1$, the frequency of the laser field is comparable to the tunnel frequency of the electron, hence tunnel ionization is the most prominent mechanism. Conversely, when $\gamma \ll 1$, the laser field comparable to the Coulomb field between the nucleus and the electron, therefore classical FI dominates. To determine how well the Keldysh

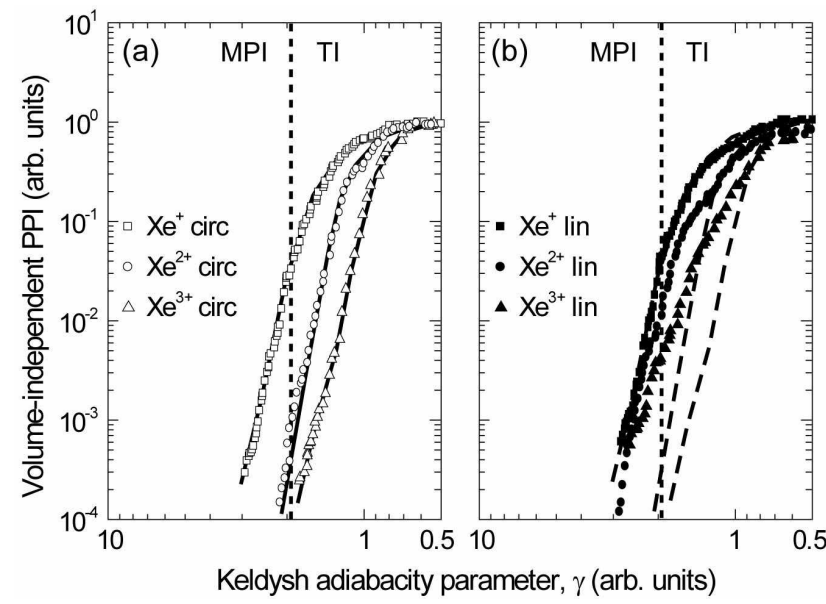

FIG. 5: PPI as a function of Keldysh parameter for $\mathrm{Xe}^{n+}(n$ $=1,2,3)$ for (a) circular and (b) linear polarization. The presence of multiphoton ionization (MPI) dominates at high $\gamma$, whereas tunnel ionization (TI) is more prominent at $\gamma<2$. A five point running average (solid line) through the points in (a) is duplicated onto (b) (dashed line) to illustrate the sequential ionization PPI as a function of $\gamma$. Interestingly, the contribution from recollision ionization in the linear pulse tends to MPI/TI response of $\mathrm{Xe}^{+}$.

adiabaticity parameter quantifies ultrafast ionization of xenon, our quantification of the ionization mechanism as presented in Fig. 4 is converted to adiabaticity in Fig. 5, and the data normalized to unity at $\gamma=0.1$.

In Fig. [5), the PPI to $\mathrm{Xe}^{n+}(n=1,2,3)$ in a circularly polarized laser pulse as a function of $\gamma$ is presented. Interestingly, the transition between MPI and TI for $n=$ 1 and $n=2$ occurs at the same $\gamma=2$, thus the identification of the presence of MPI at low intensities in $\mathrm{Xe}^{2+}$ is realistic. The vertical dashed line indicates the transition between MPI and TI occurring at $\gamma=2$, which is consistent with the intensities at which the transition between MPI and TI is observed for $\mathrm{Xe}^{+}$and $\mathrm{Xe}^{2+}$ (see Fig. 4). The position of this transition is consistent with the prediction of Keldysh. Furthermore, the PPI of $\mathrm{Xe}^{n+}(n=$ $1,2,3)$ all tend to unity at $\gamma=0.5$. For $\gamma<0.5$, FI dominates for $(n=1,2,3)$. Fig. 5(a) also contains a 5 -point running average through the experimental points (solid lines), to indicate the trend of the data. These trends are duplicated onto Fig. 5(b) as dashed lines, allowing the comparison of PPI in circular and linear polarized laser pulses.

As is apparent from Fig. 1 (b), the PPI of $\mathrm{Xe}^{n+}(n=1$, 2,3 ) in a linearly polarized laser pulse as a function of $\gamma$ is rather different from the circular case, as expected from Fig. 3 and 4 due to the presence of RI. When $n=1$, there is little deviation between linear and circular over the full range of $\gamma$ recorded. However, for $n=2$, around $\gamma=1.2$, the PPI breaks away from the response observed in the circular case (long dashed line), and tends to follow the PPI response for $n=1$. This transition, due to RI, has not been observed in this manner before. Importantly, 
the $n=1 \mathrm{PPI}$ is the upper limit of PPI enhancement by RI for $n=2$. As is apparent from Fig. 5(b), the PPI as a function of $\gamma$ for $n=3$ exhibits a similar behaviour, however as there are now two RI processes present, as $\gamma$ is decreased, the PPI for $n=3$ tends first to the PPI for $n=2$ around $\gamma=1.3$, then around $\gamma=2.3$, tends to the $n=1$ response.

\section{SUMMARY}

A new investigation into atomic ionization dynamics has been presented, employing a novel experimental technique allowing a direct comparison between linear and circular polarizations. The key to effective intensity matching (EIM) is defining the spatial dependence of ionization yield as independent of polarization type. Intensity selective scanning (ISS) is used to measure the ionization of xenon as a function of laser intensity by translating the focusing optic with respect to a spatially limited time-of-flight spectrometer.

By deriving a solution for the diffraction of a Gaussian laser pulse through an arbitrary ABCD optical system, geometric effects have been removed from the EIMISS results, producing partial probabilities of ionization (PPI) for $\mathrm{Xe}^{n+}(n=1,2,3)$ for both linear and circular polarizations. This technique has allowed clear measurement of the PPI due to recollision ionization, which contributes significantly to double and triple PPI in a linear polarized laser field. Multiphoton, tunnel and field ionization contributions are clearly identified for the charge states presented for both linear and circular polarizations. We find that for the lowest intensities, multiphoton ionization successfully predicts ionization to $\mathrm{Xe}^{+}$and $\mathrm{Xe}^{2+}$. As the laser intensity is increased, tunnel ionization theory is extremely successful, up to the intensity at which classical field ionization dominates.

The volume-independent PPIs have also been presented as a function of the Keldysh adiabaticity parameter for linear and circular polarizations. The applicability of multiphoton and tunnel ionization theory allows a precise definition of the transition between ionization mechanisms, which occurs when the Keldysh adiabaticity parameter, $\gamma=2$. Furthermore, an interesting dependence on the lower ionization states is observed for recollision ionization in a linearly polarized laser pulse. This due to the necessity to generate the source ion before recollision can proceed.

\section{Acknowledgments}

This research was supported by the Engineering and Physical Sciences Research Council (EPSRC), UK: both EMLE and SLW acknowledge studentships from the EPSRC, JMK would also like to acknowledge funding from DEL, MS from IRCEP at QUB.

\section{APPENDIX A: DIFFRACTION QUANTIFICATION}

The conventional approach for treating the propagation of light beams is through the solution of the generalized Huygens-Fresnel integral (see for example [34]). Specifically, the Collins form [35] of this integral is often used, as it allows the ABCD system matrix to be directly incorporated into the calculation. When the propagation of the beam is limited by some form of aperture, solving the Huygens-Fresnel integral analytically becomes more difficult, and it often requires a numerical solution. The difficulty then becomes one of generating a sufficiently accurate, computationally efficient solution. This has been addressed recently by Lü and Ji [14], who deftly adapted an approximation of the aperture function of Wen and Breazeale 15. to allow the analytical treatment of multiapertured ABCD optical systems for the first time. However, the approximate aperture function employed introduces oscillations into the light amplitude even before any diffraction effects are present, thus it is felt that a more accurate solution to the problem of propagating Gaussian beams through apertured ABCD optical systems is warranted.

\section{General solution}

In considering the Huygens-Fresnel integral [34] for an arbitrary ABCD system matrix in polar co-ordinates, the beam intensity on the output plane $U\left(r_{2}, \phi, z\right)$ is defined as

$U\left(r_{2}, \phi, z\right)=\frac{1}{\lambda B} \int_{0}^{2 \pi} \int_{0}^{\infty} U_{0}\left(r_{1}, \theta, z=0\right) \exp (\mathrm{i} k S) r_{1} d r_{1} d \theta$

also referred to as the Collins diffraction integral [35], where

$$
S=z+\frac{1}{2 B}\left(A r_{1}^{2}-2 r_{1} r_{2} \cos [\theta-\phi]+D r_{2}^{2}\right)
$$

is the path between point $\left(r_{1}, \theta\right)$ on the input plane and point $\left(r_{2}, \phi\right)$ on the output plane, and $z$ is the distance between the input and output planes. Variables $A, B$ and $D$ are the elements of the system matrix. For a Gaussian input beam, in the plane defined by $r_{1}$ and $z=0$, we define

$$
U_{0}\left(r_{1}, \theta, z=0\right)=\exp \left(-r_{1}^{2} / \omega_{g}^{2}\right)
$$

where $\omega_{g}$ defines the beam radius in the input plane. By substituting Eq. (A3) and Eq. (A2) into Eq. (A1), and employing the following expression from laser resonator calculations 36 .

$$
\begin{array}{r}
\int_{0}^{2 \pi} \exp \left[\mathrm{i}\left(\frac{k r_{1} r_{2}}{X} \cos (\theta-\phi)-l \theta\right)\right] d \theta \\
=2 \pi \mathrm{i}^{l} \exp (-\mathrm{i} l \phi) J_{l}\left(\frac{k r_{1} r_{2}}{X}\right)
\end{array}
$$


where $J_{l}$ is a Bessel function of the first kind and $l^{\text {th }}$ order, we arrive at the following general expression for $l$ $=0$ :

$$
\begin{aligned}
& U\left(r_{2}, \phi, z\right)=\frac{2 \pi}{\lambda B} \exp \left(\frac{\mathrm{i} k D r_{2}^{2}}{2 B}\right) \exp (\mathrm{i} k z) \\
& \times \int_{0}^{\infty} \exp \left(P r_{1}^{2}\right) J_{0}\left(Q r_{1}\right) r_{1} d r_{1}
\end{aligned}
$$

where

$$
\begin{aligned}
& P=-\frac{1}{\omega_{g}^{2}}+\frac{\mathrm{i} k A}{2 B} \\
& Q=\frac{k r_{2}}{B}
\end{aligned}
$$

and $k=2 \pi / \lambda$. The analytical solution of this integral is now examined for the unapertured and apertured cases. It is assumed that the ABCD optical system generates a focus in the vicinity of the output plane, however the solutions presented apply for any system matrix.

\section{Unapertured solutions}

Many contemporary laser-dilute matter experiments assume an unapertured Gaussian pulse profile, which when focused generates the following well-known distribution of intensity, $I\left(r_{2}, z\right)$

$$
I\left(r_{2}, z\right)=\frac{I_{0}}{1+\left(z / z_{0}\right)^{2}} \exp \left(\frac{-2 r_{2}^{2}}{\omega_{0}^{2}\left[I_{0} /\left[1+\left(z / z_{0}\right)^{2}\right]\right.}\right)
$$

where $r_{2}$ and $z$ define the co-ordinate frame in the vicinity of the focus, and the the beam waist, $\omega_{0}=2 f \lambda / \pi \omega_{g}$ and Rayleigh range, $z_{0}=\pi \omega_{0}^{2} / \lambda$ characterize the intensity distribution. In these expressions, $f$ is focal length of the lens generating the focus, $\lambda$ the wavelength and $\omega_{g}$ the $1 / e^{2}$ unfocussed beam diameter. The on axis distribution can be trivially found by setting $r_{2}=0$. Solving Eq. (A5) for $r_{2}=0$, the on-axis unapertured solution for the current treatment is found by evaluating the integral $\int_{0}^{\infty} \mathrm{d} r_{1}$, giving

$$
U\left(r_{2}=0, z\right)=-\frac{\pi}{\lambda B P} \exp (\mathrm{i} k z)
$$

This expression may be expanded to generate the offaxis unapertured solution, by performing the integration in Eq. (A5) as before for $\int_{0}^{\infty} \mathrm{d} r_{1}$, but with $r_{2} \neq 0$, with a solution

$$
U\left(r_{2}, z\right)=-\frac{\pi}{\lambda B P} \exp \left(\frac{\mathrm{i} k D r_{2}^{2}}{2 B}\right) \exp (\mathrm{i} k z) \exp \left(Q^{2} / 4 P\right)
$$

This solution requires the real part of $P$ to be negative, and the imaginary part of $Q$ to be zero. As this is satisfied for all cases by the definitions in Eq. (5), this is a universal solution.

\section{Apertured solutions}

To allow for the action of a finite aperture before the ABCD optical system, the integral in Eq. (A5) is evaluated $\int_{0}^{a} \mathrm{~d} r_{1}$ where $a$ is the aperture radius. The on-axis solution is found by setting $r_{2}=0$, producing the following expression

$$
U\left(r_{2}=0, z\right)=\frac{\pi}{\lambda B P} \exp (\mathrm{i} k z)\left(\exp \left(a^{2} P\right)-1\right)
$$

where the conditions applied to Eq. (A5) are applicable here.

To find a solution to the off-axis apertured system, the integral in Eq. (A5) is evaluated $\int_{0}^{a} \mathrm{~d} r_{1}$ where $a$ is the aperture radius. This equation cannot be solved analytically, so two methods of calculating the spatial intensity distribution are employed. Firstly, we perform a Taylor Series expansion around $r_{2}=0$, of the integral term in Eq. (A5), so the general form is thus

$$
\begin{aligned}
U\left(r_{2}, z\right) & =\frac{2 \pi}{\lambda B} \exp \left[\mathrm{i} k\left(\frac{D r_{2}^{2}}{2 B}+z\right)\right] \\
& \times \sum_{y=0}^{\infty} \sum_{x=0}^{y}\left(\frac{-1^{y}}{(y-x) !} \frac{P^{y-x} Q^{2 x}}{2^{2 x}(x !)^{2}}\right)
\end{aligned}
$$

where $x$ and $y$ are the indices of the expansion terms. This solution can be used to calculate the distribution of intensity for certain optical systems depending on $P$, $Q$ and $a$. For a relatively sharp focus ( $f$ of the order of $\left.\omega_{g}\right)$ with little diffraction $\left(a \gg \omega_{g}\right)$, the number of terms required in the Taylor Series is small. However, when the system exceeds these limits, particularly when there is significant diffraction of the incoming beam, the number of terms required becomes untenable, and the $(x !)^{2}$ term requires impracticable computational power to evaluate. In this case, the intensity distribution may be evaluated by converting to a finite element problem through

$$
\begin{aligned}
U\left(r_{2}, z\right) & =\frac{2 \pi}{\lambda B} \exp \left[\mathrm{i} k\left(\frac{D r_{2}^{2}}{2 B}+z\right)\right] \\
& \times \sum_{r_{1}=0}^{a} r_{1} \exp \left(P r_{1}^{2}\right) J_{0}\left(Q r_{1}\right) \Delta r_{1}
\end{aligned}
$$

This solution requires accurate and rapid evaluation of the Bessel Function, which becomes processor-intensive as $Q r_{1}$ becomes large. By approximating $J_{n}\left(Q r_{1}\right) \simeq$ $\left(2 / \pi Q r_{1}\right)^{1 / 2} \cos \left[Q r_{1}-(n \pi / 2)-(\pi / 4)\right]$ when $Q r_{1}>8$, evaluation of this sum becomes very efficient while retaining a deviation from the expected value of $<1$ in $10^{4}$. The off-axis solution is therefore 


$$
U\left(r_{2}, z\right)=\frac{2 \pi}{\lambda B} \exp \left[\mathrm{i} k\left(\frac{D r_{2}^{2}}{2 B}+z\right)\right] \sum_{r_{1}=0}^{a} r_{1} \exp \left(P r_{1}^{2}\right)\left(\frac{2}{\pi r_{1} Q}\right)^{1 / 2} \cos \left(Q r_{1}-\frac{\pi}{4}\right) \Delta r_{1}
$$

By making $\Delta r_{1}$ small, Eq. (A11) and (A12) will produce an extremely accurate quantification of the output plane. The selection of which form to employ depends on the geometry of the ABCD system and the size of the incoming beam relative to the aperture diameter. Computational efficiency may be further improved through the application of Adaptive Mesh Refinement 37], where the grid spacing depends on the rate of variation in $U$ resolved along both the $r_{2}$ and $z$ axes.
An important test for the off-axis apertured solution, Eq. (A10) to Eq. (A12) is to check for convergence to the off-axis unapertured solution, Eq. (A8) and the accepted definition, Eq. (A6) when $a \gg \omega_{g}$. Through rigorous two-dimensional comparisons for a variety of ABCD systems, beam radius $\omega_{g}$ and aperture radius $a$ have shown that all solutions presented are self-consistent, and, when applicable, consistent with the accepted solution.
[1] M Ferray, A. L'Huillier, X. F. Li, L. A. Lompré, G. Mainfray and C. Manus, J. Phys. B 21 L31 (1988)

[2] M. Hentschel et al, Nature (London) 414509 (2001), R. Kienberger et al, Nature (London) 427817 (2004).

[3] K. J. Schafer, B. Yang, L. F. DiMauro and K. C. Kulander, Phys. Rev. Lett. 701599 (1993), P. B. Corkum, Phys. Rev. Lett. 711994 (1993)

[4] C. Cornaggia and Ph. Hering, Phys. Rev. A 62023403 (2000)

[5] J. H. Posthumus, Rep. Prog. Phys. 67623 (2004).

[6] J. B. Greenwood, I. M. G. Johnston, P. McKenna, I. . Williams, T. R. J. Goodworth, J. H. Sanderson, W. A. Bryan, A. El-Zein, W. R. Newell, A. J. Langley and E. J. Divall, Phys. Rev. Lett. 88233001 (2002).

[7] S. L. Stebbings et al, submitted to J. Phys. B.

[8] M. Suresh, J. McKenna, B. Srigengan, I. D. Williams, E. M. L. English, S. L. Stebbings, W. A. Bryan, W. R. Newell, E. J. Divall, C. J. Hooker and A. J. Langley, Nucl. Instrum. and Meth. in Phys. Res. B 235216 (2005)

[9] S. Backus, C. G. Durfee III, M. M. Murnane and H. C. Kapteyn, Rev. Sci. Instrum. 691207 (1998)

[10] M. A. Walker, P. Hansch and L. D. Van Woerkom, Phys. Rev. A 57 R701 (1998).

[11] P. Hansch, M. A. Walker and L. D. Van Woerkom, Phys. Rev. A (54 R2559 (1996).

[12] C. Guo, M. Li, J. P. Nibarger and G. N. Gibson, Phys. Rev. A 58 R4271 (1998)

[13] T. R. J. Goodworth, W. A. Bryan, I. D. Williams and W. R. Newell, J. Phys. B: At. Mol. Opt. Phys. 383083 (2005).

[14] B. Lü and X. Ji, J. Opt. A: Pure Appl. Opt. 6161 (2004).

[15] J. J. Wen and M. A. Breazeale, J. Acoust. Soc. Am. 83 1752 (1988).

[16] L. Zhang, L. J. Frasinski and K. Codling, J. Phys. B: At. Mol. Opt. Phys. 273427 (1994).

[17] K. Kondo, A. Sagisaka, A. Tamida, Y. Nabekawa and S. Watanabe, Phys. Rev. A 48 R2531 (1993)

[18] B. Walker, B. Sheehy, L. F. DiMauro, P. A. Agostini, K. J. Schafer and K. C. Kulander, Phys. Rev. Lett. 731227 (1994)

[19] L. F. DiMauro and P. Agostini, Adv. At. Mol. Opt. Phys.
3579 (1995)

[20] A. Talebpour, C. Y. Chein, Y. Liang, S. Larochelle and S. L. Chin, J. Phys. B: At. Mol. Opt. Phys. 301721 (1997)

[21] S. Larochelle, A. Talebpour and S. L. Chin, J. Phys. B: At. Mol. Opt. Phys. 311201 (1998)

[22] B. Sheehy, R. Lafon, M. Widmer, B. Walker, L. F. DiMauro, P. Agostini and K. C. Kulander, Phys. Rev. A 583942 (1998)

[23] L. V. Keldysh, Sov. Phys. JEPT 201307 (1965).

[24] W. A. Bryan, J. H. Sanderson, A. El-Zein, W. R. Newell, P. F. Taday and A. J. Langley, J. Phys. B: At. Mol. Opt. Phys. 33745 (2000)

[25] V. L. B. de Jesus, B. Feuerstein, K. Zorst, D. Fischer, A. Rudenko, F. Afanch, C. D. Schröter, R. Moshammer and J. Ullrich, J. Phys. B: At. Mol. Opt. Phys. 37 L161 (2004)

[26] T. Auguste, P. Monot, L. A. Lompré, G. Mainfray and C. Manus, J. Phys. B: At. Mol. Opt. Phys. 254181 (1992)

[27] E. B. Tulenko and B. A. Zon, Laser Phys. 13450 (2003).

[28] A. M. Perelomov, V. S. Popov and M. V. Terent'ev, Sov. Phys. JETP 23924 (1966), 24207 (1967), A. M. Perelomov and V. S. Popov, Sov. Phys. JETP 2542 (1967).

[29] P. B. Corkum, Phys. Rev. Lett. 711994 (1993).

[30] H. G. Muller, P. Agostini and G. Petite, in Atoms in intense laser fields, edited by M. Gavrila, Adv. At. Mol. Opt. Phys., Academic Press (1992).

[31] K. Yamakawa, Y. Akahane, Y. Fukuda, M. Aoyama, N. Inoue, H. Ueda and T. Utsumi, Phys. Rev. Lett. 92 123001 (2004).

[32] A. Becker, R. Dörner and R. Moshammer, J. Phys. B: At. Mol. Opt. Phys. 38 S753 (2005)

[33] See, for example, R. Moshammer et al, Phys. Rev. Lett. 84, 447 (2000).

[34] M. Born and E. Wolf, Principles of Optics, 5th ed. New York: Pergamon Press (1975).

[35] S. A. Collins, J. Acoust. Soc. Am. 601168 (1970).

[36] J. P. Taché, Appl. Opt. 262698 (1987).

[37] M. Berger and J. Oliger, J. Comput. Phys. 53484 (1984), M. Berger and P. Colella, J. Comput. Phys. 8262 (1989). 